Joerg C. Schefold*, Linn Porz, Barbara Uebe, Holger Poehlmannª, Stephan von Haehling, Andreas Jung, Nadine Unterwalder and Christian Meisel

\title{
Diminished HLA-DR expression on monocyte and dendritic cell subsets indicating impairment of cellular immunity in pre-term neonates: a prospective observational analysis
}

\begin{abstract}
Aims: The risk of neonates for severe infection/sepsis is reciprocally proportional to gestational age and birth weight. As monocytes and dendritic cells (DC) are recognised key antigen-presenting immune cells, we aimed to elucidate whether neonatal age is associated with reduced expression of human-leukocyte antigen-DR (HLA-DR) antigens on subsets of monocytes and DCs.

Methods: Forty-three consecutive neonates (20 male, mean gestational age 236.0 \pm 26.8 days; mean 1-min Apgar score $7.5 \pm 2.0$ ) were included in a monocentric prospective observational analysis. Patients were grouped according to gestational age ( $\mathrm{n}=15$ full-term, $\mathrm{n}=28$ pre-term defined as $<33$ weeks). Ten healthy adult volunteers were assessed also. Flow-cytometric assessment of HLA-DR expression was performed in subsets of peripheral blood myeloid and
\end{abstract}

a Deceased.

*Corresponding author: Joerg C. Schefold, MD, Department of Intensive Care Medicine, University Hospital of Bern, Inselspital CH 3010 Bern, Switzerland, Phone: +41-31-63 25397, E-mail: joerg.schefold@insel.ch; and Department of Nephrology and Intensive Care Medicine, Charité Universitätsmedizin Berlin, Campus Vichow Clinic, Augustenburger Platz 1, Berlin, Germany Linn Porz and Holger Poehlmann: Department of Medical Immunology, Charité Universitätsmedizin Berlin, Campus Virchow Clinic, Augustenburger Platz 1, Berlin, Germany

Barbara Uebe and Andreas Jung: Department of Neonatology, Charité Universitätsmedizin Berlin, Campus Charité Mitte, Charitéplatz 1, Berlin, Germany

Stephan von Haehling: Department of Clinical Cardiology, Charité Universitätsmedizin Berlin, Campus Virchow Clinic, Augustenburger Platz 1, Berlin, Germany; and Department of Cardiology and Pneumology, Universitätsmedizin Göttingen, Göttingen, Germany Nadine Unterwalder: Department of Immunology, Labor Berlin Charité Vivantes, Sylter Strasse 2, Berlin, Germany Christian Meisel: Department of Medical Immunology, Charité Universitätsmedizin Berlin, Campus Virchow Clinic, Augustenburger Platz 1, Berlin, Germany; and Department of Immunology, Labor Berlin Charité Vivantes, Sylter Strasse 2, Berlin, Germany
plasmacytoidDCs (MDCand PDC) and monocytes (CD14 ${ }^{\text {bright }}$ CD16 ${ }^{\text {negative }} / \mathrm{CD} 14^{\text {positive }}$ CD $\left.16^{\text {positive }} / \mathrm{CD} 14^{\text {dim }} \mathrm{CD} 16^{\text {positive }}\right)$. Clinical and routine laboratory data were followed up.

Results: At birth, leukocyte counts were increased in full-term neonates. Monocyte counts were significantly increased in neonates when compared with adults (all $\mathrm{P}<0.05)$. A significant numerical increase of $\mathrm{CD} 14^{\text {bright }}$ $\mathrm{CD} 16^{\text {negative }}$ and $\mathrm{CD} 14^{\text {positive }} \mathrm{CD} 16^{\text {positive }}$ monocytes was noted in pre-term and full-term neonates (all $\mathrm{P}<0.05$ ), while HLA-DR expression in these subsets was significantly diminished (most pronounced in pre-term infants, $\mathrm{P}<0.0001)$. MDC and PDC HLA-DR expression was reduced also (all $\mathrm{P}<0.05$ ). Clinical indices (e.g., $\mathrm{pH}$, days on antibiotics/mechanical ventilation, fever/sepsis) were not found to correlate with immunological indices.

Conclusions: We observed a markedly diminished HLADR expression on monocyte and DC subsets in pre-term and full-term neonates, which may contribute to impaired antimicrobial defence mechanisms in the early days of life.

Keywords: Dendritic cells; HLA-DR; immunoparalysis; inflammation; monocytes; organ failure; sepsis; septic shock; severe infection.

DOI 10.1515/jpm-2014-0226

Received July 15, 2014. Accepted September 26, 2014

\section{Introduction}

Severe infection or sepsis is more often observed in neonates with low gestational age or low weight at birth. This seems especially evident in very pre-term $(<28.0$ weeks of gestational age) or very low weight (i.e., $<1500$ g) neonates. Here, risks of infection and mortality rates are especially high [1-5]. It is currently acknowledged that not only organ-specific complications (e.g., intracranial 
bleedings, bronchopulmonary dysplasia) contribute to increased rates of infection, but cellular immune function may also be immature. Interestingly, immune response to vaccination in the first year of life is markedly diminished in pre-term when compared with full-term neonates of same postnatal age. This may indicate a profound and persisting immunodeficiency in pre-term neonates in early life [1, 4-6]. However, data on functional immunity in this population are scarce, and underlying immunological mechanisms are incompletely understood.

The host response to invasive microbial infection includes several crucial "defence lines" of innate and acquired immunity. In adults, numerous immune dysfunctions were reported in sepsis including altered cytokine expression profiles, immune cell apoptosis, and diminished expression of major histocompatibility (MHC) class II molecules on antigen-presenting cells (APC) [7-9]. Previously, we and others could demonstrate that diminished monocytic expression of the MHC class II molecule human-leukocyte antigen-DR (HLA-DR) reflects diminished immune cellular functionality and often occurs in late sepsis [7]. Low monocytic HLA-DR (mHLA-DR) expression was found to indicate patients at risk to develop infectious complications after major trauma, burn, or surgery [10]. In sepsis, persistent diminished mHLA-DR was also termed sepsis-associated immunosuppression (SAI) and is associated with adverse outcome. Clinical pilot trials aiming at SAI reversal showed that this can be achieved by both immunostimulation and reduction of systemic inhibitors [11, 12]. Nevertheless, whether or not SAI reversal improves clinical outcomes from sepsis is currently investigated.

In neonates, monocytes appear at weeks 18-20 in the circulation, and a constant physiological increase in total numbers occurs until birth $[1-5,10]$. Consequently, preterm neonates mostly have diminished monocyte counts $[1,4,5,13,14]$. However, in contrast to the extensively studied major population of classical CD14 ${ }^{\text {bright }}$ monocytes, little knowledge exists on Fc $\gamma$ receptor III positive (i.e., CD16 $\left.{ }^{\text {bright }}\right)$ monocytes in neonates. This subpopulation was referred to as "pro-inflammatory monocytes" $(-10 \%$ of circulating monocytes in adults) and expresses higher pro-inflammatory stimulation-induced cytokine levels. HLA-DR expression of CD14 ${ }^{\text {bright }} \mathrm{CD} 16^{\text {negative }}$ monocytes has been shown reduced in pre-term neonates.

Dendritic cells (DCs) are considered the most potent APCs and link innate and adaptive immune responses [15]. Mature DCs typically express high numbers of HLA-DR molecules. Two major DC phenotype subsets can be distinguished in peripheral blood: myeloid (MDC) and plasmacytoid DCs (PDC). These DC subsets are functionally different in regard to mode of activation, cytokine release, and effector function. Activation of MDCs by microbial products (e.g., lipopolysaccharides) results in the secretion of pro-inflammatory cytokines (e.g., tumor necrosis factor- $\alpha$ ), whereas PDCs produce large amounts of interferon- $\alpha$ and enhance antiviral defence. Interestingly, animal sepsis models and human data indicate that compromised DC function is associated with adverse outcomes [16].

In an effort to better understand the immune pathophysiology, we investigate phenotypic differences in monocyte and DC subsets in pre-term and full-term neonates.

\section{Materials and methods}

\section{Population and protocol}

In a tertiary-care academic centre, a total of 43 consecutive neonates were included in a monocentric prospective observational analysis. Between December 2005 and July 2007, n=28 pre-term (defined as $<33$ weeks of gestational age; "pre-term group") neonates and $\mathrm{n}=15$ full-term neonates (defined as $>36$ weeks; "full-term group") were included. Ten healthy adult volunteers were assessed also (comparison group, aged $27.7 \pm 3.92$ years, means \pm SD). The observational analysis was performed at the Department of Neonatology, Charité University Hospital, Campus Mitte, Berlin, Germany. Inclusion criteria were the following: gestational age according to groups "pre-term" and "full-term" (see above). Exclusion criteria were the following: denial of consent by parents, pre-existing chromosomal anomalies, innate congenital atypical infections such as cytomegalovirus/rubella/parvovirus B19 infection, HIV positivity, and indication of toxoplasmosis or tuberculosis (TBC). Informed consent was achieved from parents/legal representatives. The study was performed in adherence to the Declaration of Helsinki and approved by the local ethics committee on human research.

\section{Points in time of clinical and laboratory assessment}

Patients were assessed at the following points in time: T1: 72-96 h post partum, T2: $96-120 \mathrm{~h}$ post partum, T3: day 11 ( \pm 3 days), T4: day 18 ( \pm 3 days), T5: day 25 ( \pm 3 days). Full-term neonates and adult controls were assessed at a single time point only (T1 for full-term neonates). Comparison of data from the full term/pre-term group with the data derived from samples of healthy adults was performed at this point in time only. Clinical data including age, ethnicity, gestational/morphological age, twin/multiple birth, mode of delivery (e.g., caesarean section), weight, Apgar score, Clinical Risk Index for Babies (CRIB) score, $\mathrm{FiO}_{2}$, vital signs, temperature, and antibiotic medication were recorded. If available, the following laboratory indices were assessed: $\mathrm{pH}$ at birth, haematocrit, haemoglobin concentration, total bilirubin levels, C-reactive protein, platelet counts, and total white blood cell (WBC)/monocyte count. Quantification of 
immune phenotypic markers was performed in the certified immunological laboratory (Institute of Medical Immunology, Charité University Hospital, Berlin, Germany).

\section{Quantification of HLA-DR expression and analysis of DC subsets}

Quantification of HLA-DR on circulating monocytes was performed using a standardised flow cytometric assay (QuantiBRITE HLA-DR, BD Biosciences, Heidelberg, Germany), as described elsewhere [17]. Enumeration of DC subsets was performed as described elsewhere [16]. In short, $150 \mu \mathrm{L}$ of whole blood was stained with flourescein isothiocyanate-conjugated antibodies against lineage (lin1) markers (mixture of anti-CD3/CD14/CD16/CD19/CD20/CD56), anti-CD123-PE, anti-HLA-DR-PerCP, and anti-CD33-APC (BD Biosciences, Heidelberg, Germany). PDC were gated as lin1- CD123+ HLA-DR+ events and MDC as lin1- CD33+ HLA-DR+. After treatment with FACS Lysing Solution, at least 300 events per DC population were analyzed on a FACSCalibur using CellQuest ${ }^{\text {Pro }}$ (BD Biosciences, Heidelberg, Germany) software. HLA-DR expression on DCs was measured as mean fluorescence intensity. Absolute APC population frequencies were calculated as WBC counts multiplied by the ratio of the APC population over all leukocytes. The gating strategy is given (Figure 1).

\section{Statistical analysis}

For statistical analyses, MedCalc 12.0 (MedCalc, Mariakerke, Belgium) was used. Data are given as mean \pm SD, if not indicated otherwise. Data were checked for normal distribution using the Kolmogorov-Smirnov test; for summary data, please refer to respective analysis. Patients who fulfilled the entry criteria including provision of informed consent entered the analysis. An initial formal power analysis/sample size calculation was not performed. Observation of effects was considered the focal point. $\mathrm{P}<0.05$ was considered significant.

\section{Results}

\section{Study population and follow-up}

Study population details are given in Table 1. A total of $\mathrm{n}=43$ patients (20 male, mean gestational age $236.0 \pm 26.8$ days; mean 1-min Apgar score 7.5 \pm 2.0 ) were included. Distribution of gender, ethnicity, and baseline $\mathrm{pH}$ did not differ between the pre-term and full-term groups. As expected, significant between-group differences were noted in gestational age, morphological age, and weight at birth (all $\mathrm{P}<0.0001$ ). The mode of delivery differed between groups, with a higher prevalence of caesarian section and twin/multiple births in the pre-term group (Table 1). Apgar scores differed significantly also. At 1, 5, and 10 min, significantly lower Apgar scores were noted in pre-term individuals. In the pre-term group, $n=2$ patients developed severe sepsis/septic shock, and a total of $n=7$ developed infant/acute respiratory distress syndrome (IRDS/ARDS). Patients in the pre-term group received mechanical ventilation for $8.0 \pm 9.4$ days (mean \pm SD), and antibiotic treatment was performed for $2.8 \pm 4.7$ days (Table 1). All patients survived. Overall, we regard the two groups to reflect typical neonate cohorts treated at a tertiary-care academic centre. The comparison group $(n=10)$ consisted of healthy laboratory personnel (aged $27.7 \pm 3.9$ years at the time the trial was ended).

\section{Distribution of leukocytes and monocytes subsets in neonates}

According to respective CD14 and CD16 surface expressions, three distinct monocyte subsets can be identified in the peripheral blood: "classical" monocytes (i.e., $\left.\mathrm{CD} 14^{\text {bright }} \mathrm{CD} 16^{\text {negative }}\right)$, "pro-inflammatory" monocytes (CD14 $\left.{ }^{\text {negative }} C D 16^{\text {positive }}\right)$, and "transition" monocytes (CD14 ${ }^{\text {positive }} \mathrm{CD} 16^{\text {positive }}$ ). At baseline, it was found that both total circulating WBC counts and the total number of circulating monocytes were increased in both neonate groups (all $\mathrm{P}<0.05$ except $\mathrm{WBC}$ in pre-term individuals) when compared with adults (Figure $2 \mathrm{~A}$ and $\mathrm{B}$, respectively). Numbers of $\mathrm{CD} 14^{\text {bright }} \mathrm{CD} 16^{\text {negative }}$ monocytes were increased in both pre-term and full-term neonates when compared with controls. Statistically significant differences were not noted between the pre-term and full-term neonate groups (Figure 2C). Circulating levels of CD14 $4^{\text {posi- }}$ ${ }^{\text {tive }} \mathrm{CD} 16^{\text {positive }}$ monocytes were found significantly increased in both pre-term and full-term neonates when compared with adults (Figure 2D). The total number of $\mathrm{CD} 14^{\mathrm{dim}} \mathrm{CD}$ $16^{\text {positive }}$ monocytes was elevated in full-term but not preterm neonates when compared with adults (Figure 2E).

\section{Distribution of dendritic cells (subsets) in neonates}

Human peripheral blood contains at least two distinct populations of DCs. In the current prospective investigational analysis, the number and function of PDC (i.e., lineage CD123+ HLA-DR+ DCs) and MDC (i.e., lineage- CD33+ HLA$\mathrm{DR}+\mathrm{DCs}$ ) were investigated. Compared with adult healthy controls, the MDC numbers were reduced in neonates (5.3 \pm 3.0 and $6.1 \pm 4.5 / \mu \mathrm{L}$ for pre-term/full-term neonates, respectively) when compared with adults $(13.0 \pm 4.4 / \mu \mathrm{L})$. The number of PDCs were comparable in the groups 

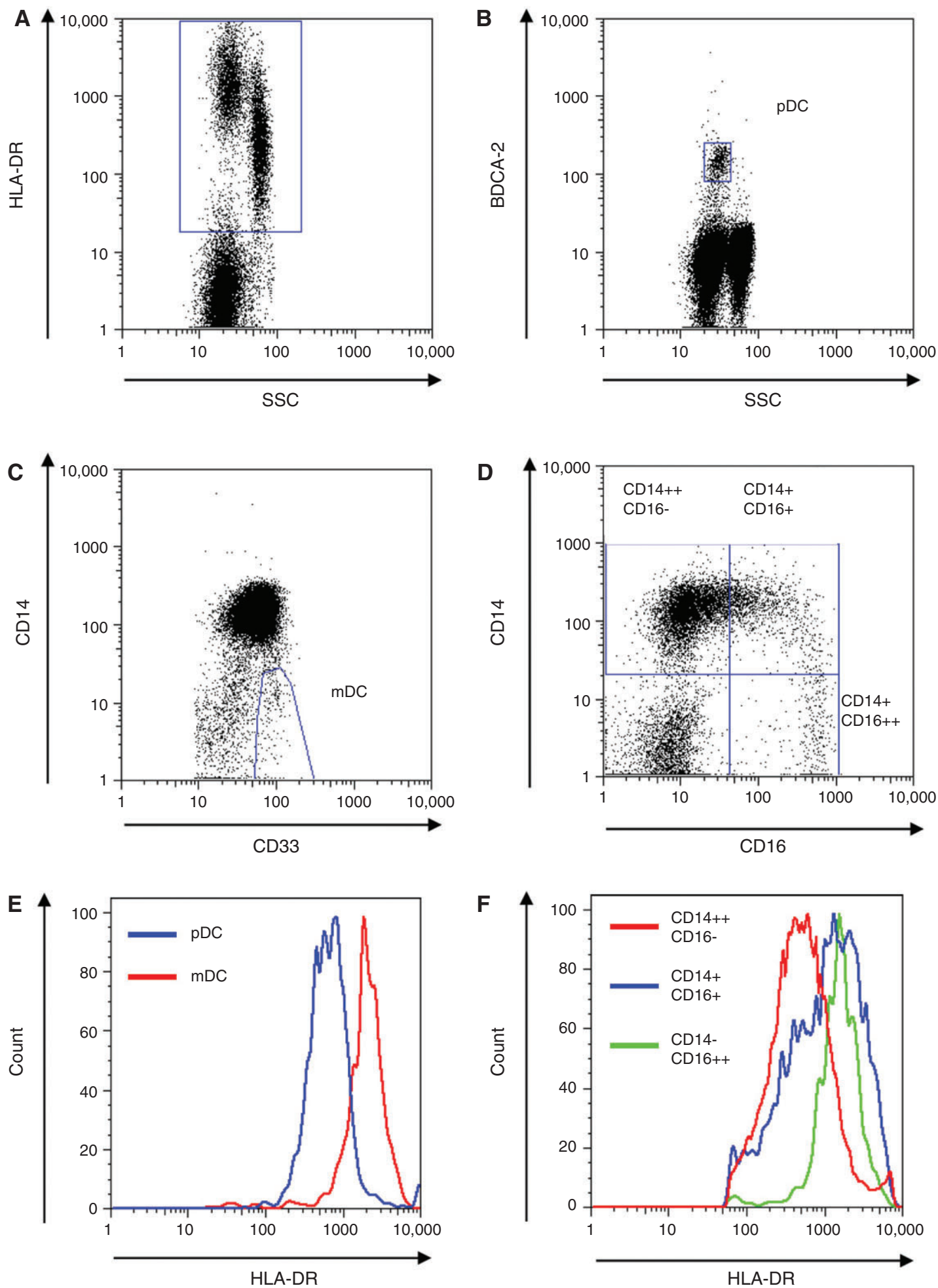

Figure 1 Gating strategy and HLA-DR expression on dendritic cell (DC) and monocyte subsets. Mononuclear cells were gated using a forward scatter/side scatter (SSC) dot plot, and HLA-DR positive cells were identified using a HLA-DR/SCC dot plot (A). Plasmacytoid DC (PDC) were defined as HLA-DR+ BDCA-2+ cells using a BDCA-2/SSC dot plot (B), and myeloid DC (MDC) were identified as CD14- CD33++ cells using a CD14/CD33 dot plot (C). According to their differential expression of CD14/CD16, three monocyte subsets were defined: CD14++CD16+, CD14+CD16+, and CD14+/-CD16++ (D). Examples of HLA-DR expression on DC and monocyte subsets are given in the histogram plots (E) and (F). 
Table 1 Baseline demographics, disease severity, and clinical follow-up.

\begin{tabular}{|c|c|c|c|}
\hline & $\begin{array}{r}\text { Pre-term } \\
\text { group }(n=28)\end{array}$ & $\begin{array}{r}\text { Full-term } \\
\text { group }(n=15)\end{array}$ & $\begin{array}{l}\text { Between- } \\
\text { group P-value }\end{array}$ \\
\hline Gestational age (days) & $218.4 \pm 11.5$ & $268.9 \pm 10.7$ & $<0.0001$ \\
\hline Gender (male) & $11(39 \%)$ & $9(60 \%)$ & $=0.22^{\mathrm{a}}$ \\
\hline \multicolumn{4}{|l|}{ Morphological age } \\
\hline (weeks) & $31.6 \pm 1.6$ & $38.1 \pm 1.4$ & $<0.0001$ \\
\hline (days) & $221.2 \pm 10.9$ & $266.9 \pm 9.9$ & $<0.0001$ \\
\hline Body weight (g) & $1448 \pm 336$ & $3346 \pm 430$ & $<0.0001$ \\
\hline Baseline blood pH (at delivery, venous blood sample) & $7.3 \pm 0.1$ & $7.3 \pm 0.1$ & 0.30 \\
\hline Ethnicity (Caucasian patients) & $23(82 \%)$ & $15(100 \%)$ & $0.14^{\mathrm{a}}$ \\
\hline Delivery mode (caesarean section) & $21(75 \%)$ & $5(33 \%)$ & $0.01^{\mathrm{a}}$ \\
\hline Twin birth/multiple birth & $13(46 \%)$ & $1(6 \%)$ & $0.01^{\mathrm{a}}$ \\
\hline Development of severe sepsis/septic shock (follow-up period) & $2(7 \%)$ & $0(0 \%)$ & $0.54^{a}$ \\
\hline Development of respiratory distress syndrome (follow-up period) & $7(25 \%)$ & $0(0 \%)$ & $0.08^{\mathrm{a}}$ \\
\hline \multicolumn{4}{|l|}{ Apgar score } \\
\hline At 1 min post partum & $6.9 \pm 2.1$ & $8.6 \pm 1.5$ & 0.01 \\
\hline At 5 min post partum & $7.9 \pm 1.4$ & $9.2 \pm 0.8$ & 0.001 \\
\hline At 10 min post partum & $8.7 \pm 0.7$ & $9.5 \pm 0.7$ & 0.002 \\
\hline \multicolumn{4}{|l|}{ CRIB score } \\
\hline Total CRIB score at birth & $1.0 \pm 1.1$ & - & - \\
\hline CRIB value: birth weight & $0.6 \pm 0.8$ & - & - \\
\hline CRIB value: gestational age (weeks) & $0.0 \pm 0.0$ & - & - \\
\hline CRIB value: congenital dysplasia & $0.1 \pm 0.3$ & - & - \\
\hline CRIB value: maximum base deficit (12 h) & $0.2 \pm 0.7$ & - & - \\
\hline \multicolumn{4}{|l|}{ Clinical follow-up } \\
\hline Days on mechanical ventilator & $8.0 \pm 9.4$ & - & - \\
\hline Days on vasopressors & $0.04 \pm 0.2$ & - & - \\
\hline Days on antibiotics & $2.8 \pm 4.7$ & - & - \\
\hline Days with central venous line & $1.9 \pm 4.5$ & - & - \\
\hline Days with apnoea episodes(in 28 days) & $3.1 \pm 4.4$ & - & - \\
\hline Days with fever ( $>38^{\circ}$, within 28 days) & $0.7 \pm 1.2$ & - & - \\
\hline Urinary catheterisation (days) & $0.4 \pm 2.1$ & - & - \\
\hline Gl tube feeding (days) & $25.8 \pm 3.6$ & - & - \\
\hline Transfusion: erythrocyte concentration (mL) & $117.8 \pm 325.0$ & - & - \\
\hline
\end{tabular}

a'Fisher's exact test was used for analysis of significance levels.

investigated $(4.7 \pm 3.4,7.7 \pm 4.1$, and $6.4 \pm 5.4 / \mu \mathrm{L}$ in pre-term neonates, full-term neonates, and adult controls).

\section{HLA-DR expression on monocyte and DC subsets}

Reduced HLA-DR expression on APC such as monocytes and DCs is a key feature of immunodepression in major trauma, burn, or sepsis. Although data exist on HLA-DR expression on $\mathrm{CD} 14^{\text {bright }} \mathrm{CD} 16^{\text {negative }}$ monocytes in neonates, only few data are available on the expression of HLA-DR in monocyte and DC subsets in pre-term and full-term neonates. This seems important as "immune function" is not only a consequence of normal levels of circulating respective immune cells but also a consequence of immune functionality. HLA-DR expression on $\mathrm{CD} 14^{\text {bright }} \mathrm{CD} 16^{\text {negative }}$ and $\mathrm{CD} 14^{\text {positive }} \mathrm{CD} 16^{\text {positive }}$ monocytes (in Ab/cell) was strongly reduced in neonates, and this effect was most pronounced in pre-term individuals (Figure 3A and B). Interestingly, this effect was not observed in $\mathrm{CD} 14^{\text {negative }} \mathrm{CD} 16^{\text {positive }}$ monocytes (Figure 3C). In this subgroup, HLA-DR expression was enhanced in full-term infants, while HLA-DR expression in pre-term infants was comparable with that of adult controls (Figure 3C). When compared with adults, baseline HLA-DR expression on both MDC (Figure 3D) and PDC (Figure 3E) was reduced in both pre-term and full-term neonates.

\section{Course of immune function (monocytes and DC subsets) in neonates}

To assess the course of immune function in pre-term newborns, we assessed the expression of HLA-DR in monocytes and DC cells over time (Table 2). HLA-DR significantly 

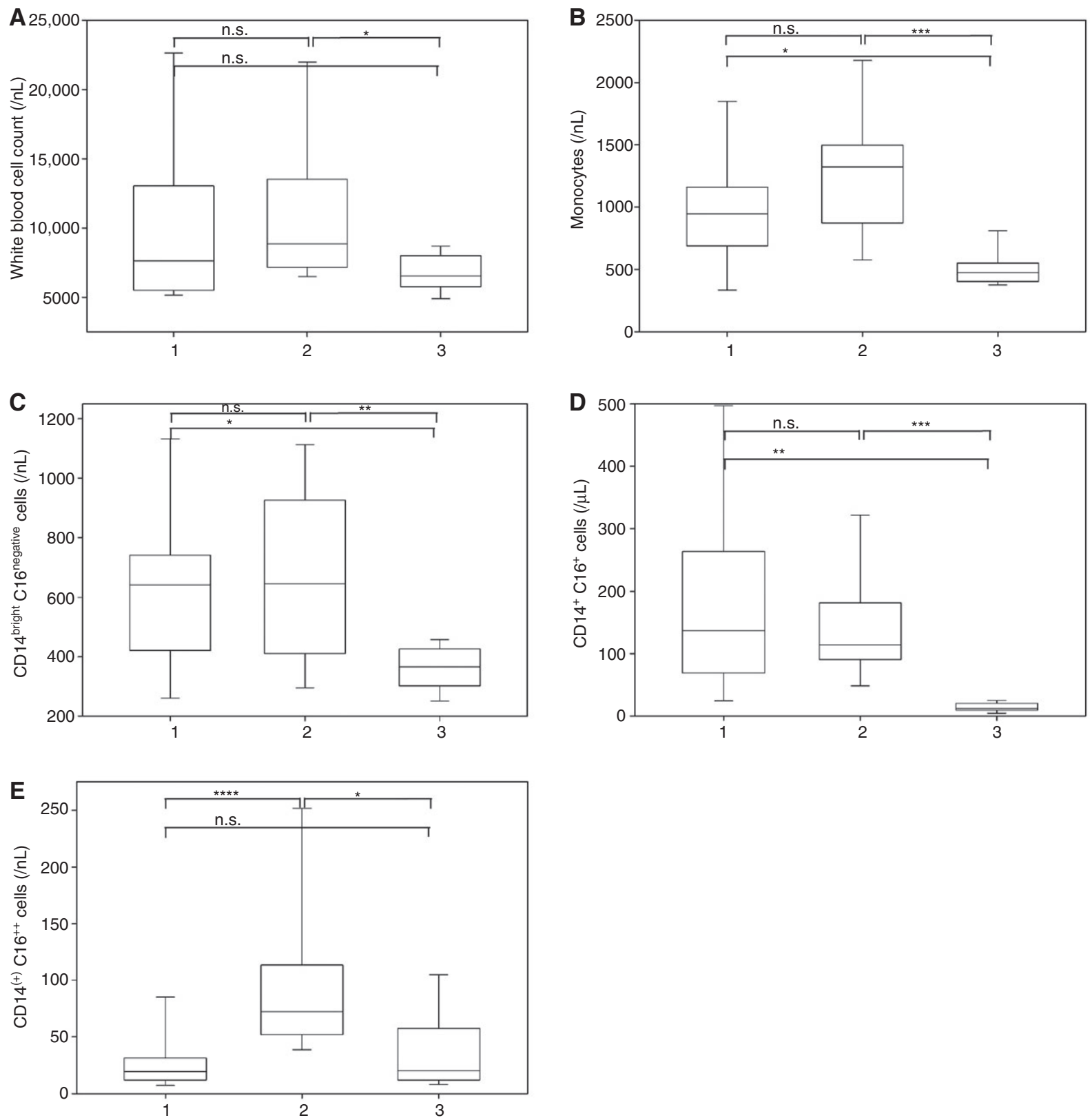

Figure 2 The absolute number of circulating immune cells in pre-term (group 1) and full-term (group 2) neonates at baseline is given (cells $/ \mu \mathrm{L}$ ) and compared with the number of circulating cells in adult healthy controls (control group: group 3). (A) Total number of circulating white blood cells (WBC), (B) total number of circulating monocytes, (C) total number of circulating CD14 ${ }^{\text {bright }}$ CD16 ${ }^{\text {negative }}$ ("classical") monocytes, (D) total number of circulating CD14 ${ }^{\text {positive }}$ CD16 $6^{\text {positive }}$ ("transition") monocytes, and (E) total number of circulating

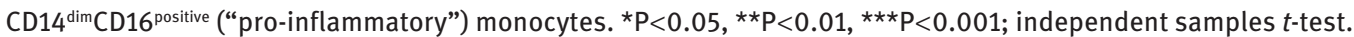

increased on all three monocyte subsets from $\mathrm{T} 1$ to $\mathrm{T} 5$ and thus tended towards normalisation/the levels observed in adult healthy controls (Table 2). With regard to DCs, it was noted that the HLA-DR expression on MDCs increased over time, reaching levels comparable with levels of adult controls on T4/T5. Interestingly, the level of HLA-DR on PDCs remained unchanged over the observational interval (Table 2).

\section{Correlation of HLA-DR expression with clinical indices}

A significant correlation of gestational age with the degree of mHLA-DR expression on all three subsets was noted: increased gestational age was strongly associated with increased mHLA-DR expression $(\mathrm{P}<0.001$ for all comparisons). In DCs, this relation was not noted $(\mathrm{P}=0.85$ and $\mathrm{P}=0.1$ 

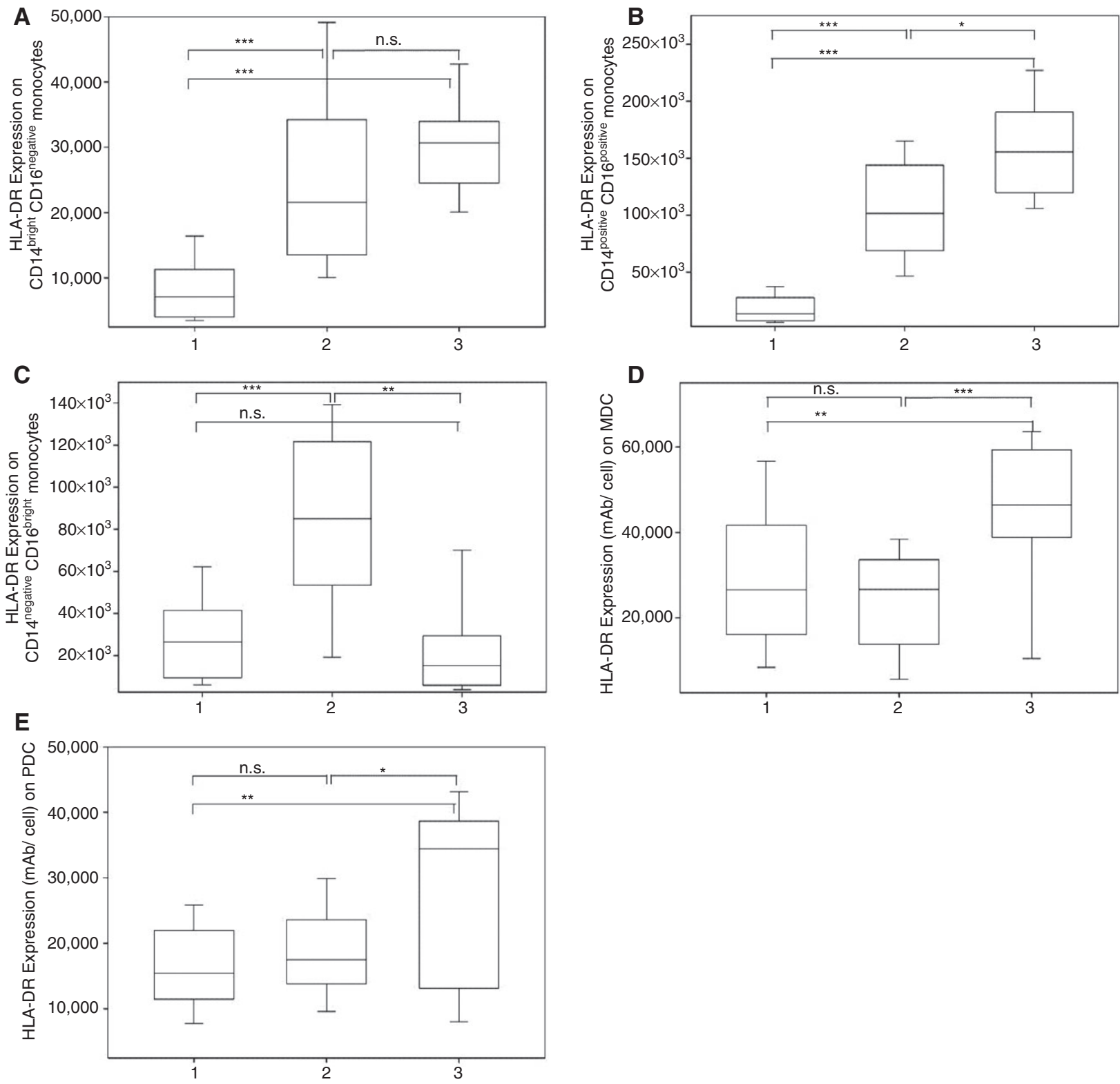

Figure 3 Baseline HLA-DR expression on respective monocyte and DC subsets (given in mAb/cell) in pre-term (group 1) and full-term (group 2) neonates compared with adult healthy controls (group 3). (A) circulating CD14 ${ }^{\text {bright }} C D 16^{\text {negative }}$ ("classical") monocytes, (B) CD14 ${ }^{\text {positive }}$ CD-

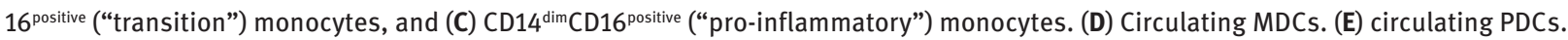
${ }^{*} \mathrm{P}<0.01,{ }^{\star \star} \mathrm{P}<0.001,{ }^{* \star *} \mathrm{P}<0.0001$; independent samples $t$-test.

for MDCs and PDCs, respectively). Interestingly, reduced weight at birth was significantly associated with diminished HLA-DR expression in CD14 $4^{\text {bright }} C D 16^{\text {negative }}$, CD14 $4^{\text {positive }}$ $\mathrm{CD} 16^{\text {positive }}$, and $\mathrm{CD} 14^{\text {negative }} \mathrm{CD} 16^{\text {bright }}$ monocytes $(\mathrm{P}<0.001$ for all comparisons), whereas this was not noted in MDCs and PDCs ( $\mathrm{P}=0.44$ and $\mathrm{P}=0.35$, respectively). Moreover, no association was observed between $\mathrm{pH}$ at birth and the expression of HLA-DR on monocytes or DCs in both pre-term and full-term neonates. Interestingly, we found a strong association of 1-min Apgar scores with mHLA-DR expression (all $\mathrm{P}<0.02$ ). Furthermore, we investigated whether total days on antibiotics was associated with HLA-DR expression of monocytes or DCs. Antibiotics were given in 16 patients, and mean total days on antibiotics was $2.8 \pm 4.7$ days. No significant association between total days on antibiotics or total days on mechanical ventilation (28-day observational period) with monocytic/DC HLA-DR expression was found (all $\mathrm{P}>0.2$ ). The number of apnoea episodes and days with hyperthermia $\left(>38.0^{\circ} \mathrm{C}\right)$ were not associated with monocytic or DC HLA-DR expression. 


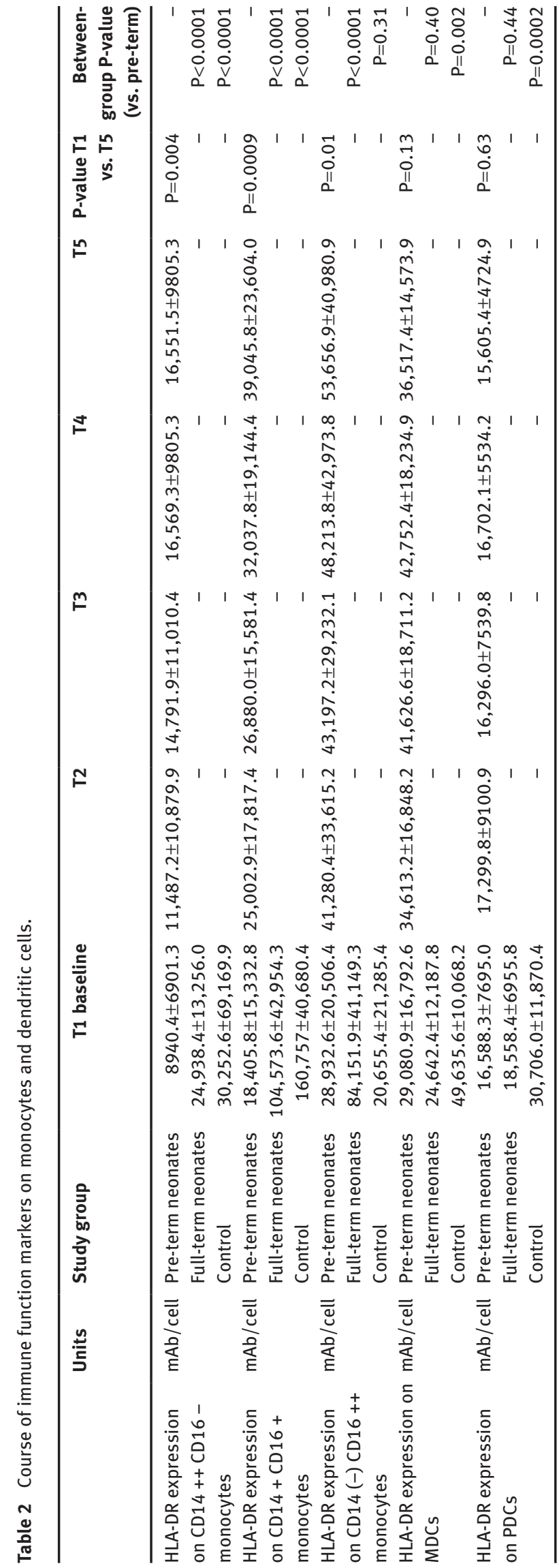

\section{Group of patients with respiratory distress}

In seven infants (all pre-term), respiratory distress syndrome was noted. In this group, gestational age was significantly reduced (210.6 \pm 17.1 days (IRDS group) vs. $241.2 \pm 25.9$ days (group without IRDS), $\mathrm{P}=0.0005$ ). Weight at birth was significantly reduced in this group also (1442.9 $\pm 407.4 \mathrm{~g}$ (IRDS group) vs. 2257.1 $\pm 1024.7 \mathrm{~g}$ (group without IRDS), $\mathrm{P}=0.05)$. HLA-DR on $\mathrm{CD} 14^{\text {bright }} \mathrm{CD} 16^{\text {negative }}$ and $\mathrm{CD} 14^{\text {positive }} \mathrm{CD} 16^{\text {positive }}$ monocytes was significantly down-regulated in patients with IRDS $(5285 \pm 3128$ vs. $16,239 \pm 12,403 \mathrm{mAb} /$ cell for CD14 ${ }^{\text {bright }} \mathrm{CD} 16^{\text {negative }}$ monocytes $(\mathrm{P}=0.03)$ and $11,986 \pm 3255$ vs. $54,865 \pm 51,386 \mathrm{mAb} /$ cell for $\mathrm{CD} 14^{\text {positive }} \mathrm{CD} 16^{\text {positive }}$ monocytes $(\mathrm{P}=0.04)$, respectively). Given the limited number of IRDS cases, respective data must be interpreted with caution. A multifactorial analysis was therefore not performed.

\section{Discussion}

Severe infection is a serious and life-threatening condition especially in pre-term neonates. The risk of pre-term neonates to acquire severe infection or sepsis is reciprocally proportional to gestational age and birth weight. As monocytes and DCs are recognised key antigen-presenting immune cells, we aimed to elucidate whether diminished gestational age is associated with reduced expression of HLA-DR antigens on monocyte and DC subsets. This was performed in order to better understand the complex immune pathophysiology in the neonatal age.

We observed that at birth, total leukocyte and monocyte counts were found increased when compared with adults, a previously described fact that could at least partially be attributable to stress. When subgroups of monocytes were investigated $\left(\mathrm{CD} 14^{\text {bright }} \mathrm{CD} 16^{\text {negative }}, \mathrm{CD} 14^{\text {positive }}\right.$ $\mathrm{CD} 16^{\text {positive }}$, and $\mathrm{CD} 14^{\mathrm{dim}} \mathrm{CD} 16^{\text {positive }}$ ), we observed that the total number of $\mathrm{CD} 14^{\text {bright }} \mathrm{CD} 16^{\text {negative }}$ and $\mathrm{CD} 14^{\text {positive }} \mathrm{CD} 16^{\text {positive }}$ monocytes was increased in pre-term and full-term newborns. Interestingly, this effect was not observed in pre-term infants in the monocyte subset referred to as "pro-inflammatory" (CD14 $\left.{ }^{\mathrm{dim}} \mathrm{CD} 16^{\text {positive }}\right)$, whereas respective levels were increased in full-term infants. Although we are unable to elucidate the mechanisms behind this, it seems tempting to speculate that reduced total numbers of $\mathrm{CD} 14^{\text {dim }} \mathrm{CD} 16^{\text {positive }}$ monocytes might indicate an additional lack of immunocompetence. Moreover, we investigated the number of MDCs and PDCs in the respective study groups. At baseline, PDCs were rather unchanged between the three study groups, whereas MDCs were 
found reduced in pre-term and full-term infants compared with adults.

As protective immune reponses depend on effective antigen presentation and induction of adaptive immune mechanisms, we investigated cellular immune function also. To assess cellular immune function, the quantitative surface HLA-DR expression was assessed on monocyte and DC subsets. A major reduction in HLA-DR expression in $\mathrm{CD} 14^{\text {bright }} \mathrm{CD} 16^{\text {negative }}$ and $\mathrm{CD} 14^{\text {positive }} \mathrm{CD} 16^{\text {positive }}$ monocytes in newborns was noted. Before the background of increased total cell numbers, this indicates functional deactivation rather than numerical down-regulation. In addition to respective cell counts of $\mathrm{CD} 14^{\mathrm{dim}} \mathrm{CD} 16^{\text {positive }}$ monocytes, HLA-DR expression on this specific subpopulation of monocytes was unchanged. This may indicate that functional deactivation of monocytes mainly occurs in "classical" rather than "pro-inflammatory" monocytes. In addition, DC subsets (i.e., MDC and PDC) showed diminished HLA-DR surface expression. However, when compared with monocyte subsets, this effect was less pronounced.

From a clinical perspective, we were interested whether the observed immunological changes were associated with clinical parameters of immaturity. We found that lower gestational age and birth weight was significantly associated with diminished mHLA-DR expression ( $\mathrm{P}<0.001$ for all subsets), whereas this was not observed in MDCs and PDCs. However, venous $\mathrm{pH}$ at birth, days on antibiotics or mechanical ventilation, total number of apnoea episodes, development of hyperthermia/sepsis, and Apgar scores were not found to correlate with respective immunological indices.

There are a number of limitations of our analysis that seem important to discuss. First, the number of study patients investigated was rather limited and the overall recruitment period was rather long. This implies an obvious major limitation of our analysis and, by definition, we cannot rule out an effect of low sample size on our findings. Moreover, a formal sample size calculation or power analysis was not performed. This was due to the observational proof-of-concept character of the analysis. Importantly, we are therefore unable to investigate, e.g., gender-related differences, differences induced by the mode of delivery, or other clinical endpoints/effects. Second, provision of informed consent by respective legal proxies was necessary and patients were deliberately recruited on a consecutive basis. By definition, this might have introduced a bias to the analysis also. Third, due to restrictions in blood volume, we were able to investigate a limited set of immune parameters only. Fourth, we were unable to systematically access a potential influence of the respective mothers' immune status on the immune system of respective newborns. This was beyond the scope of the analysis and should be investigated in subsequent analyses. Fifth, we are unable to conclude on the underlying pathomechanism of decreased MHC class II expression on APC as this was beyond the scope of our analysis.

\section{Conclusion}

In conclusion, we demonstrate a profound and sustained functional impairment of circulating $\mathrm{CD} 14^{\text {bright }} \mathrm{CD} 16^{\text {negative }}$ and $\mathrm{CD} 14^{\text {positive }} \mathrm{CD} 16^{\text {positive }}$ monocytes in pre-term and fullterm newborns. This state of functional immunological immaturity may contribute to impaired antimicrobial defence mechanisms. Further studies are required to elucidate the individual role of distinct APC populations in neonatal infection/sepsis and whether functional APC reconstitution may prevent infectious complications and improve outcomes.

Acknowledgments: The authors thank all ICU and laboratory staff for their dedicated help and support.

Competing interests: The authors declare that they have no competing interests to declare.

Authors' contributions: JCS and CM designed the trial, investigated all data, and wrote the manuscript. LP, HP, and $\mathrm{AJ}$ were responsible for patient recruitment and patient management, recorded all data, and assisted in the interpretation of all data. SVH participated together with JCS and CM in the interpretation and statistical analysis of all data and revised the manuscript for important intellectual content.

\section{References}

[1] Adkins B, Leclerc C, Marshall-Clarke S. Neonatal adaptive immunity comes of age. Nat Rev Immunol. 2004;4:553-64.

[2] Christensen RD, Anstall HB, Rothstein G. Review: deficiencies in the neutrophil system of newborn infants, and the use of leukocyte transfusions in the treatment of neonatal sepsis. J Clin Apher. 1982;1:33-41.

[3] Gentile LF, Nacionales DC, Lopez MC, Vanzant E, Cuenca A, Cuenca AG, et al. Protective immunity and defects in the neonatal and elderly immune response to sepsis. J Immunol. 2014 (epub ahead of print).

[4] Kovarik J, Siegrist CA. Immunity in early life. Immunol Today. 1998;19:150-2. 
[5] Velilla PA, Rugeles MT, Chougnet CA. Defective antigenpresenting cell function in human neonates. Clin Immunol. 2006;121:251-9.

[6] Andrews P, Azoulay E, Antonelli M, Brochard L, Brun-Buisson C, Dobb G, et al. Year in review in intensive care medicine, 2004. III. Outcome, ICU organisation, scoring, quality of life, ethics, psychological problems and communication in the ICU, immunity and hemodynamics during sepsis, pediatric and neonatal critical care, experimental studies. Intensive Care Med. 2005;31:356-72.

[7] Hotchkiss RS, Monneret G, Payen D. Sepsis-induced immunosuppression: from cellular dysfunctions to immunotherapy. Nat Rev Immunol. 2013;13:862-74.

[8] Schefold JC, Hasper D, Reinke P, Monneret G, Volk HD. Consider delayed immunosuppression into the concept of sepsis. Crit Care Med. 2008;36:3118.

[9] Schefold JC, Hasper D, Volk HD, Reinke P. Sepsis: time has come to focus on the later stages. Med Hypotheses. 2008;71:203-8.

[10] Meisel C, Schwab JM, Prass K, Meisel A, Dirnagl U. Central nervous system injury-induced immune deficiency syndrome. Nat Rev Neurosci. 2005;6:775-86.

[11] Meisel C, Schefold JC, Pschowski R, Baumann T, Hetzger K, Gregor J, et al. Granulocyte-macrophage colony-stimulating factor to reverse sepsis-associated immunosuppression: a double-blind, randomized, placebo-controlled multicenter trial. Am J Respir Crit Care Med. 2009;180:640-8.
[12] Schefold JC, von Haehling S, Corsepius M, Pohle C, Kruschke P, Zuckermann $\mathrm{H}$, et al. A novel selective extracorporeal intervention in sepsis: immunoadsorption of endotoxin, interleukin 6, and complement-activating product 5 a. Shock. 2007;28:418-25.

[13] Gladstone IM, Ehrenkranz RA, Edberg SC, Baltimore RS. A tenyear review of neonatal sepsis and comparison with the previous fifty-year experience. Pediatr Infect Dis J. 1990;9:819-25.

[14] Hallwirth U, Pomberger G, Pollak A, Roth E, Spittler A. Monocyte switch in neonates: high phagocytic capacity and low HLADR expression in VLBWI are inverted during gestational aging. Pediatr Allergy Immunol. 2004;15:513-6.

[15] Huang X, Venet F, Chung CS, Lomas-Neira J, Ayala A. Changes in dendritic cell function in the immune response to sepsis. Cell- \& tissue-based therapy. Expert Opin Biol Ther. 2007;7:929-38.

[16] Poehlmann H, Schefold JC, Zuckermann-Becker H, Volk HD, Meisel C. Phenotype changes and impaired function of dendritic cell subsets in patients with sepsis: a prospective observational analysis. Crit Care. 2009;13:R119.

[17] Docke WD, Hoflich C, Davis KA, Rottgers K, Meisel C, Kiefer P, et al. Monitoring temporary immunodepression by flow cytometric measurement of monocytic HLA-DR expression: a multicenter standardized study. Clin Chem. 2005;51:2341-7.

The authors stated that there are no conflicts of interest regarding the publication of this article. 